\title{
Time-varying spectral analysis for comparison of HRV and PPG variability during tilt table test
}

\author{
Eduardo Gil, Michele Orini, Raquel Bailón, José María Vergara, Luca Mainardi and Pablo Laguna
}

\begin{abstract}
In this work we assessed the possibility of using the pulse rate variability (PRV) extracted from photoplethysmography signal as an alternative measurement of the HRV signal in non-stationary conditions. The study is based on the analysis of the changes observed during tilt table test in the heart rate modulation of 17 young subjects. Time-varying spectral properties of both signals were compared by time-frequency (TF) and TF coherence analysis. In addition, the effect of replacing PRV with HRV in the assessment of the changes of the autonomic modulation of the heart rate was considered. Timefrequency analysis revealed that: the TF spectra of both signals were highly correlated $(0.99 \pm 0.01)$; the difference between the instantaneous power, in LF and HF bands, obtained from HRV and PRV was small $\left(<10^{-3} \mathrm{~s}^{-2}\right)$ and their temporal patterns were highly correlated $(0.98 \pm 0.04$ and $0.95 \pm 0.06$ in $L F$ and HF bands respectively); TF coherence in $\mathrm{LF}$ and $\mathrm{HF}$ bands was high $(0.97 \pm 0.04$ and $0.89 \pm 0.08$, respectively). Finally, the instantaneous power in LF band was observed to significantly increase during head-up tilt by both HRV and PRV analysis. These results suggest that, although some small differences in the time-varying spectral indices extracted from HRV and PRV exist, mainly in the HF band associated with respiration, PRV could be used as an acceptable surrogate of HRV during nonstationary conditions, at least during tilt table test.
\end{abstract}

\section{INTRODUCTION}

Heart rate variability (HRV) analysis is one of the most widely used non-invasive techniques for the evaluation of the autonomic nervous system. Power spectral density of the HRV exhibits oscillations related to the parasympathetic and sympathetic activities [1]. The range between 0.003 and 0.04 $\mathrm{Hz}$ (very low-frequency component, VLF) takes account of long term regulation mechanisms. The range between 0.04 and $0.15 \mathrm{~Hz}$ (low-frequency component, LF) represents both sympathetic and parasympathetic modulation, although an increase in its power is generally associated with a sympathetic activation. The range between 0.15 and $0.4 \mathrm{~Hz}$ (highfrequency component, HF) corresponds to parasympathetic modulation and is synchronous with the respiratory rate.

Pulse photoplethysmography (PPG) is a simple and useful method for measuring the relative blood volume changes in

This work was partially supported by Ministerio de Ciencia y Tecnología, FEDER under Project TEC2007-68076-C02-02/TCM and Grupo Consolidado GTC from DGA.

E. Gil, M. Orini, R. Bailón and P. Laguna are with Communications Technology Group, Aragón Institute of Engineering Research, University of Zaragoza, Zaragoza, Spain and CIBER de Bioingeniería, Biomateriales y Nanomedicina (CIBER-BBN), Instituto de Salud Carlos III (FEDER).edugilheunizar.es

M. Orini and L. Mainardi are with Department of Bioengineering, Politecnico di Milano, Milano, Italy

J.M. Vergara is with Sleep Department, Miguel Servet Children Hospital, Zaragoza, Spain and CIBER de Bioingeniería, Biomateriales y Nanomedicina (CIBER-BBN), Instituto de Salud Carlos III (FEDER). the microvascular bed of peripheral tissues and evaluating peripheral circulation. PPG has been applied in many different clinical settings, including the monitoring of blood oxygen saturation, heart rate, blood pressure (BP), cardiac output and respiration. It is generally accepted that PPG can provide valuable information about the cardiovascular system. The autonomic influences on PPG signal have been analysed in several studies and recently pulse rate variability (PRV) extracted from PPG has been studied as a potential surrogate of HRV [2], [3], [4]. Since PPG is simple, has a low cost and also allows to derive physiological parameters such as blood oxygenation and ventilatory rate, the use of PRV could be particularly suitable in those applications where the simultaneous acquisition of many signals is required, as for example in sleep disorders studies. The difference between HRV and PRV is the time it takes the pulse wave to travel from the heart to the finger. This time, called the pulse transit time (PTT), is tie-related to arterial compliance and BP.

All the studies exploring the possibility of using PRV as an alternative measurement of HRV have been performed in stationary conditions using time-invariant analysis. However, there are many situations where significant changes in autonomic balance occur, as during orthostatic test, Valsalva maneuver, exercise stress testing and after pharmacologic interventions, which involve non-stationary processes. We focused this study on tilt table test. In this test, after headup tilt, subjects undergo a progressive orthostatic stress and blood pressure is maintained thanks to cardiovascular regulation, which involves an increase in heart rate and a constriction of the blood vessels in the legs. This slight tachycardia and vasoconstriction are the result of sympathetic activation and vagal withdrawal. When the supine position is restored, heart rate and vasoconstriction return to previous basal values together with sympathetic tone.

The aim of this study is to evaluate the usefulness of PRV as a surrogate of HRV analysis during non-stationary conditions, in particular, during tilt table test. Time-frequency (TF) and TF coherence analysis were performed to asses whether the PRV can be used in the analysis of the autonomic modulation of heart rate in non-stationary conditions.

\section{MATERIAL AND METHODS}

\section{A. Data and signal preprocessing}

Seventeen volunteers (age $28.5 \pm 2.8$ years, 11 males) underwent a head-up tilt table test according to the following protocol: 4 minutes in early supine position $\left(T_{1}\right), 5$ minutes tilted head-up to an angle of 70 degrees $\left(T_{2}\right)$ and 4 minutes back to later supine position $\left(T_{3}\right)$, see Fig. 1 . The PPG signal 


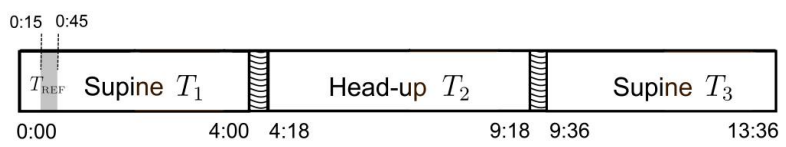

Fig. 1. Head-up tilt test protocol. Table takes $18 \mathrm{~s}$ for tilting during transitions, marked as lined area.

was recorded from index finger with a sampling frequency of $250 \mathrm{~Hz}$, whereas standard lead V4 ECG signal was recorded with a sampling frequency of $1000 \mathrm{~Hz}$ simultaneously.

Beats from ECG and pulses from PPG were detected to generate heart and pulse rate time series. The temporal location of each R wave in the ECG $\left(t_{\mathrm{E}_{j}}\right)$ was automatically determined using the algorithm described in [5]. The PPG signal was interpolated using cubic splines to increase the resolution in time up to an equivalent sampling rate of $1000 \mathrm{~Hz}$. Then, the temporal location of each pulse wave in the PPG $\left(t_{\mathrm{P}_{j}}\right)$ was detected as the maximum of the PPG signal within the interval $\left[t_{\mathrm{E}_{j}}+150 \mathrm{~ms}, t_{\mathrm{E}_{j+1}}\right]$. In addition, a PPG artefact detector [6] was applied to suppress pulses from PPG corresponding to artefacts and beat and pulse detections were manually supervised. Then, the effect of abnormal beats in both heart and pulse rate was corrected by applying a methodology based on the integral pulse frequency modulation model [7]. Heart rate and pulse rate signals, $d_{\mathrm{HR}}(t)$ and $d_{\mathrm{PR}}(t)$, were obtained by using a 5 th order spline interpolation at $4 \mathrm{~Hz}$ of the inverse interval function:

$$
\begin{aligned}
d_{\mathrm{IIF}}^{\mathrm{ECG}}\left(t_{\mathrm{E}_{j}}\right) & =\frac{1}{\left(t_{\mathrm{E}_{j}}-t_{\mathrm{E}_{j-1}}\right)} \\
d_{\mathrm{IIF}}^{\mathrm{PPG}}\left(t_{\mathrm{P}_{j}}\right) & =\frac{1}{\left(t_{\mathrm{P}_{j}}-t_{\mathrm{P}_{j-1}}\right)} .
\end{aligned}
$$

Finally, the HRV and PRV signals, $d_{\mathrm{HRV}}(t)$ and $d_{\mathrm{PRV}}(t)$, were calculated by high pass filtering $d_{\mathrm{HR}}(t)$ and $d_{\mathrm{PR}}(t)$ with a cutoff frequency of $0.03 \mathrm{~Hz}$.

\section{B. Time-varying analysis}

The smoothed pseudo Wigner-Ville distribution (SPWVD) was used to estimate the time-varying spectral properties of the HRV and PRV signals, as well as to perform TF coherence analysis. The cross-SPWVD of signals $x(t)$ and $y(t)$ is defined as [8]:

$$
\begin{gathered}
S_{x, y}(t, f)=\int_{-\infty}^{\infty} \int_{-\infty}^{\infty} \mathcal{K}(\tau, \nu) A_{x, y}(\tau, \nu) e^{j 2 \pi(t \nu-f \tau)} d \nu d \tau \\
A_{x, y}(\tau, \nu)=\int_{-\infty}^{\infty} x\left(t+\frac{\tau}{2}\right) y^{*}\left(t-\frac{\tau}{2}\right) e^{-j 2 \pi \nu t} d t
\end{gathered}
$$

where $A_{x, y}(\tau, \nu)$ is the narrowband symmetric ambiguity function (AF) of signals $x(t)$ and $y(t)$. The AF quantifies the TF correlation between $x(t)$ and $y(t)$ in the delaydopplerfrequency domain $(\tau, \nu)$. The kernel $\mathcal{K}(\tau, \nu)$ is a $2 \mathrm{D}$ weighting function which performs the TF low-pass filtering necessary to suppress the interference terms which reduce the readability of the Wigner-Ville distribution. Note that the SPWD of the signal $x(t)$ is obtained using $y(t)=$ $x(t)$. The analytic version of $d_{\mathrm{HRV}}(t)$ and $d_{\mathrm{PRV}}(t), a_{\mathrm{HRV}}(t)$ and $a_{\mathrm{PRV}}(t)$ respectively, were used in (3)-(4) in order to further reduce the interference terms [8]. We choose as kernel $\mathcal{K}(\tau, \nu)$ an elliptical exponential function defined as [9]:

$$
\mathcal{K}\left(\tau, \nu ; \tau_{0}, \nu_{0}\right)=\exp \left\{-\pi\left[\left(\frac{\nu}{\nu_{0}}\right)^{2}+\left(\frac{\tau}{\tau_{0}}\right)^{2}\right]^{\frac{1}{2}}\right\}
$$

Parameters $\tau_{0}$ and $\nu_{0}$ were selected to have a frequency resolution of $0.0313 \mathrm{~Hz}$ and a time resolution of $15 \mathrm{~s}$. For each subject $k$, the instantaneous power of HRV and PRV within each frequency band, $P_{\mathrm{B}}^{\mathrm{x}}(k, t)$, with $\mathrm{X} \in\{\mathrm{HRV}$, $\mathrm{PRV}\}$, was obtained integrating $S_{x}(t, f)$ in the frequency bands $\mathrm{B} \in\{\mathrm{LF}, \mathrm{HF}\}$.

The Pearson's correlation coefficient, defined as:

$\rho=\frac{C\left(\phi^{\mathrm{x}}(j), \phi^{\mathrm{Y}}(j)\right)}{\sqrt{C\left(\phi^{\mathrm{x}}(j), \phi^{\mathrm{x}}(j)\right) C\left(\phi^{\mathrm{Y}}(j), \phi^{\mathrm{Y}}(j)\right)}}, \mathrm{X}, \mathrm{Y} \in\{\mathrm{HRV}, \mathrm{PRV}\}$

was used for comparison between both sources of information. In (6), $C$ represents the covariance, $\phi(j)$ is a general parameter or signal derived from HRV or PRV and $j$ the independent variable in each case. The similarity between the temporal evolution of $d_{\mathrm{HRV}}(t)$ and $d_{\mathrm{PRV}}(t)$ was assessed by means of four indices:

1) The Pearson's correlation coefficient, $\rho_{\mathrm{I}}(k)$, between $\phi^{\mathrm{x}}(t)=P_{\mathrm{B}}^{\mathrm{HRV}}(k, t)$ and $\phi^{\mathrm{Y}}(t)=P_{\mathrm{B}}^{\mathrm{PRV}}(k, t)$ and between $\phi^{\mathrm{x}}(t)=R_{\mathrm{LF} / \mathrm{HF}}^{\mathrm{HRV}}(k, t)$ and $\phi^{\mathrm{Y}}(t)=R_{\mathrm{LF} / \mathrm{HF}}^{\mathrm{PRV}}(k, t)$.

2) The difference between the instantaneous power of the two signals within each frequency band, $\delta_{\mathrm{B}}(k, t)=$ $P_{\mathrm{B}}^{\mathrm{PRV}}(k, t)-P_{\mathrm{B}}^{\mathrm{HRV}}(k, t)$. This index is used to compare the temporal evolution of the spectral content of the signals.

3) The Pearson's correlation coefficient, $\rho_{\mathrm{s}}(k, t)$, between instantaneous spectra of the two signals is estimated at every time instant $t=t_{0}$ as in (6) with $\phi^{\mathrm{x}}(f)=$ $S_{a_{\mathrm{HRV}}}\left(t_{0}, f\right)$ and $\phi^{\mathrm{Y}}(f)=S_{a_{\mathrm{PRV}}}\left(t_{0}, f\right)$. This index is used to assess whether the signals are characterized by a similar distribution of energy with frequency.

4) The quadratic TF coherence between the two signals within each frequency band, estimated as [9]:

$\gamma^{2}(t, f)=\frac{S_{x, y}(t, f) S_{x, y}^{*}(t, f)}{S_{x}(t, f) S_{y}(t, f)} ; x, y \in\left\{a_{\mathrm{HRV}}(t), a_{\mathrm{PRV}}(t)\right\}$.

Time-frequency coherence gives a continuous quantification of spectral coherence over time, being one in epochs characterized by perfect linear coupling and zero when the two signals are completely uncorrelated. We obtained the band coherence $\gamma_{\mathrm{B}}^{2}(k, t)$ by averaging $\gamma^{2}(t, f)$ in each spectral band for each subject $k$.

\section{Physiological analysis}

In this section, we assess the effect of replacing the HRV estimation from the ECG with the PRV estimation from the PPG, when the tilt table test is used to evaluate changes in the autonomic modulation of the heart rate. We are interested in continuously monitoring these changes. To this end we quantified the statistical differences between the baseline power content $\overline{P_{\mathrm{B}}^{\mathrm{x}}}(k)$ and the instantaneous power content, $P_{\mathrm{B}}^{\mathrm{x}}(k, t)$, by iteratively performing the Student's t-test for 
every $t$. The baseline power content $\overline{P_{\mathrm{B}}^{\mathrm{x}}}(k)$ was estimated by averaging $P_{\mathrm{B}}^{\mathrm{x}}(k, t)$ in an interval $T_{\mathrm{REF}}$, selected at $T_{1}$ from 15 to $45 \mathrm{~s}$ (see Fig. 1). As result of the test we obtained a time-varying $p$-value, $p_{\mathrm{B}}^{\mathrm{x}}(t)$, for both HRV and PRV signals.

\section{RESULTS}

\section{A. Time-varying analysis}

The results of TF and TF coherence analysis for a subject (subject $k=17$, male, 30 years old) are reported in Fig. 2. Heart and pulse rates are reported in panel (a). The TF distributions of the HRV and PRV signals are shown in panels (b)-(c). $P_{\mathrm{B}}^{\mathrm{X}}(k=17, t)$, is reported in panel (d). Note that, as also shown in panels (b)-(c), the spectral properties of the HRV and PRV signals did follow the same trend. The main difference lies in the slight increase of $P_{\mathrm{HF}}^{\mathrm{PRV}}(k=17, t)$ with respect to $P_{\mathrm{HF}}^{\mathrm{HRV}}(k=17, t)$, which was more pronounced during tilt. Panel (e) shows the correlation coefficient between instantaneous spectra of the two signals, $\rho_{\mathrm{s}}(k=17, t)$. Results of the TF coherence analysis are reported in panels (f)-(g). The quadratic TF coherence $\gamma^{2}(t, f)$, reported in panel (f), shows that during $T_{1}$ and $T_{3}$ the two signals presented almost a perfect correlation for all frequencies. Around $320 \mathrm{~s}$ TF coherence decreased due to artefacts on the PPG signal (marked as crosses). Finally, the temporal evolution of the band coherence $\gamma_{\mathrm{B}}^{2}(k=17, t)$, shown in panel (g), confirms the previous observations: HRV and PRV had an almost identical TF structure, at least in LF band. It is worth noting that this is a borderline case: subject $k=17$ had one of the highest $\delta_{\mathrm{HF}}(k, t)$ and the lowest $\gamma_{\mathrm{HF}}^{2}(k, t)$ in $T_{2}$.

Next, global results, obtained by averaging among subjects the indices presented in section II-B, are reported. The correlation between the instantaneous power in each frequency band from both signals was $\rho_{\mathrm{LF}}=0.98 \pm 0.04, \rho_{\mathrm{HF}}=0.95 \pm 0.06$ and $\rho_{\mathrm{LF} H \mathrm{H}}=0.97 \pm 0.03$. Fig. 3(a)-(b) shows the mean instantaneous power within each frequency band from HRV and PRV and the corresponding instantaneous difference, respectively. Note that averaged $P_{\mathrm{B}}^{\mathrm{HRV}}(k, t)$ and $P_{\mathrm{B}}^{\mathrm{PRV}}(k, t)$ over subjects presented the same temporal patterns, even if with a bias which increased during tilt. Panel (c) shows the mean trend of the instantaneous correlation $\rho_{\mathrm{s}}(k, t)$ between the power spectral density functions derived from HRV and PRV. In the same panel we reported $\rho_{\mathrm{s}}(k=1, t)$ which corresponded to the subject who presented the highest number of artefacts in the PPG signal. It is shown that artefacts provoked an abrupt decrease in $\rho_{\mathrm{s}}(k, t)$. Panel (d) shows the band coherence $\gamma_{\mathrm{B}}^{2}(k, t)$.

\section{B. Physiological analysis results}

Fig. 4 shows that the time-varying $p$-value estimated from HRV and PRV followed the same trend, being $p_{\mathrm{LF}}^{\mathrm{HRV}}(t)$ and $p_{\mathrm{LF}}^{\mathrm{PRV}}(t)$ almost equal. The variations observed in $P_{\mathrm{LF}}^{\mathrm{x}}(k, t)$ during the tilt table test (see Fig. 3(a)) made $p_{\mathrm{LF}}^{\mathrm{x}}(t)$ change. First, immediately after the head-up tilt, $p_{\mathrm{LF}}^{\mathrm{X}}(t)$ dramatically decreased; then, during $T_{2}, p_{\mathrm{LF}}^{\mathrm{x}}(t)$ continued gradually diminishing, reaching statistical significance about 2 minutes later; finally, when the supine position was restored $p_{\mathrm{LF}}^{\mathrm{x}}(t)$ abruptly increased to previous values.

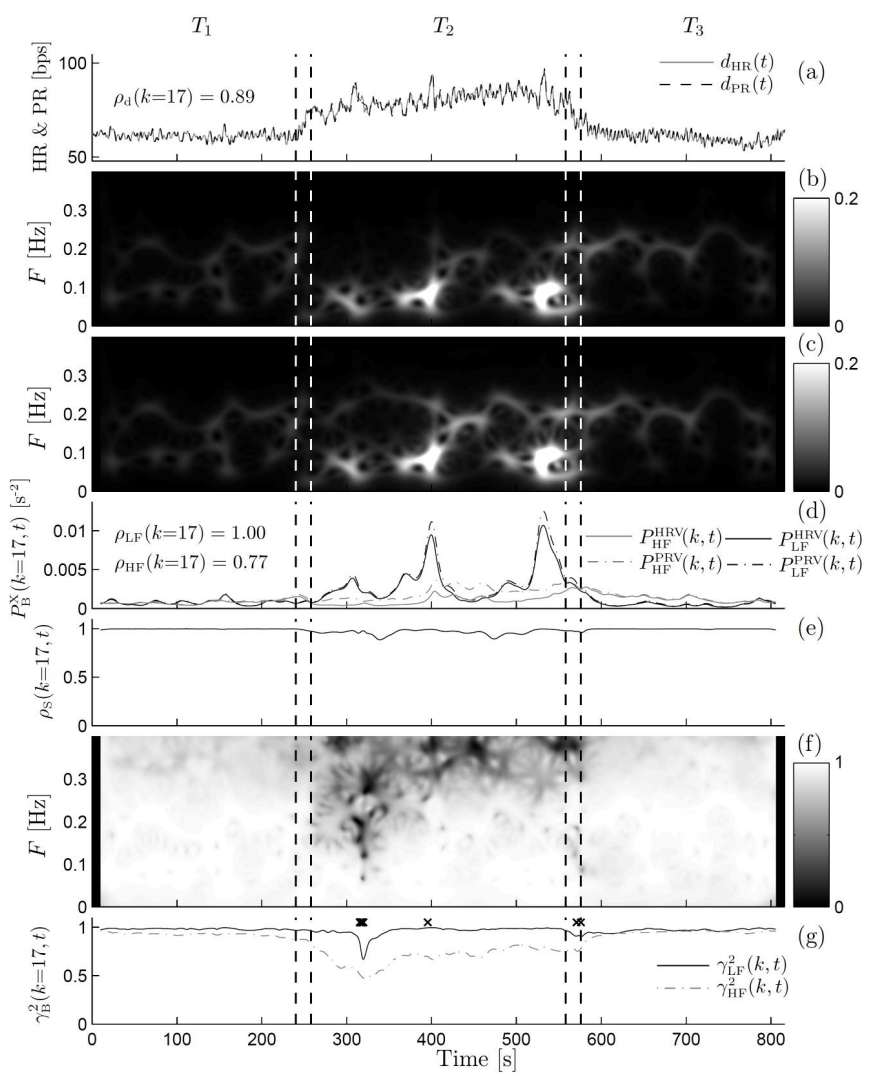

Fig. 2. (a) Heart rate and pulse rate; (b)-(c) TF distribution of $a_{\mathrm{HRV}}(t)$ and $a_{\mathrm{PRV}}(t)$, respectively; (d) $P_{\mathrm{B}}^{\mathrm{X}}(k=17, t)$ within spectral band $\mathrm{B} \in\{\mathrm{LF}, \mathrm{HF}\}$ for $\mathrm{X} \in\{\mathrm{HRV}, \mathrm{PRV}\}$; (e) Correlation coefficient between instantaneous spectra of the two signals, $\rho_{\mathrm{S}}(k=17, t)$; (f) Time-frequency coherence $\gamma^{2}(t, f)$. (g) Band coherence $\gamma_{\mathrm{B}}^{2}(k=17, t)$. Artefacts in PPG signal are marked as crosses.
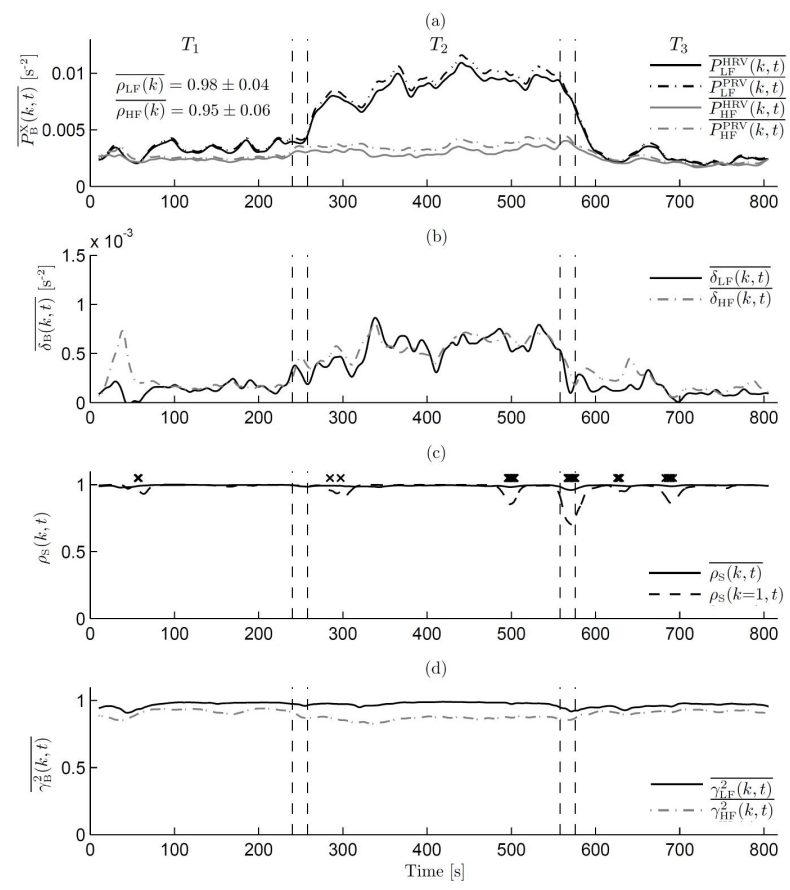

Fig. 3. Mean trend estimated by averaging among subjects. (a) $P_{\mathrm{B}}^{\mathrm{HRV}}(k, t)$ and $P_{\mathrm{B}}^{\mathrm{PRV}}(k, t)$ from HRV (continuous line) and PRV (dash-dotted line); (b) $\delta_{\mathrm{B}}(k, t)$ in LF band (grey line) and HF band (black line); (c) Instantaneous correlation $\rho_{\mathrm{S}}(k, t)$ between the power spectral density functions derived from HRV and from PRV (solid line). Index $\rho_{\mathrm{S}}(k=1, t)$ and artefacts in PPG for subject 1 are reported in dashed line and cross marks, respectively; (d) Band coherence. 
(a)

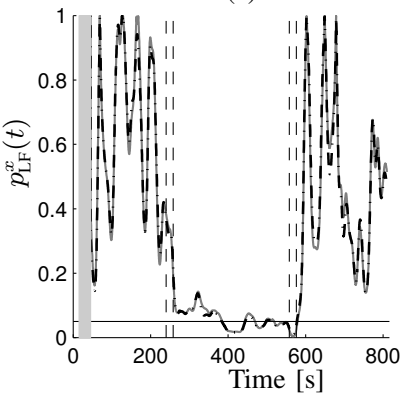

(b)

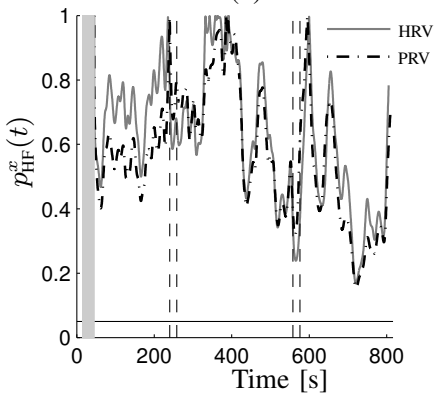

Fig. 4. Time-varying physiological analysis results. Temporal evolution of (a) the $p$-value $p_{\mathrm{LF}}^{x}(t)$ and (b) $p_{\mathrm{HF}}^{x}(t)$. Baseline values were estimated in the temporal window marked as grey area, $T_{\mathrm{REF}}$.

\section{DISCUSSION}

During tilt table test we observed high similarity between the patterns of response of the HRV and PRV signals. The global results reported in Fig. 3 show that the instantaneous power content of the PRV was slightly higher $\left(\overline{\delta_{\mathrm{B}}(k, t)}<\right.$ $\left.10^{-3} \mathrm{~s}^{-2}\right)$ than the instantaneous power content of the HRV signal. The temporal evolution of the index $\delta_{\mathrm{B}}(k, t)$ was almost the same in both frequency bands: during early and later supine positions $\overline{\delta_{\mathrm{B}}(k, t)}<0.2510^{-3} \mathrm{~s}^{-2}$, whereas during head-up position $\overline{\delta_{\mathrm{B}}(k, t)}$ increased up to a value of about $0.710^{-3} \mathrm{~s}^{-2}$. It is worth noting that during the highest non-stationary intervals (i.e. transitions where table was tilting), $\delta_{\mathrm{B}}(k, t)$ did not increase. Their temporal evolution was highly correlated, i.e. $P_{\mathrm{B}}^{\mathrm{HRV}}(k, t)$ and $P_{\mathrm{B}}^{\mathrm{PRV}}(k, t)$ followed the same trend. The correlation between the instantaneous spectral densities of the two signals, $\rho_{\mathrm{s}}(k, t)$, was also very high, being the temporal average of the mean and standard deviation among subjects $0.99 \pm 0.01$. The small decreases of $\rho_{\mathrm{s}}(k, t)$ were due to the presence of some rare artefacts in the PRV signal. The band coherence $\gamma_{\mathrm{B}}^{2}(k, t)$, showed that, despite of non-stationary conditions, the degree of linear coupling between the two signals was constant during time, and no relevant variations were observed even during upward and downward tilting. Band coherence in LF fluctuated around $0.97 \pm 0.04$ during the entire experimental procedure, while band coherence in HF fluctuated around $0.92 \pm 0.06$ and $0.87 \pm 0.10$ during supine $\left(T_{1}\right.$ and $\left.T_{3}\right)$ and head-up tilt $\left(T_{2}\right)$ position, respectively $(0.89 \pm 0.08$ during the entire experimental procedure).

We observed a positive bias in the measurement of spectral indices from PRV which increases during head-up tilt. Our hypothesis is that this could be due to the variability introduced by PTT, which could increase during head-up position. Moreover the lower values observed in $\gamma_{\mathrm{HF}}^{2}(k, t)$ with respect to $\gamma_{\mathrm{LF}}^{2}(k, t)$ suggest that, due to PTT variability, respiration is slightly differently represented in PRV than in HRV, in agreement with [10]. Spectral indices estimated from PRV and HRV analysis did follow the same temporal patterns. Nevertheless, small differences exist between their values, mainly in the respiratory band. Thus, when a study aims at accurately estimating these spectral indices, caution should be used in replacing HRV by PRV.

We observed a statistically significant increase of the power content in LF band of HRV and PRV during headup position. Simultaneous inspection of Fig. 3(a) and 4(a) reveals the transient nature of the autonomic response to orthostatic stress. It is shown that the variations in $P_{\mathrm{LF}}^{\mathrm{x}}(k, t)$ provoked changes in the temporal pattern of $p$-values. It is worth noting that there was agreement between the physiological analysis based on HRV and PRV. This suggests that PRV could be used as a surrogate measurement of HRV to evaluate the autonomic modulation changes of the heart rate during non-stationary conditions, at least in tilt table test.

It is well established that PPG measurements are quite sensitive to patient and/or probe-tissue movement artefacts. Thus the presence of motion artefacts is one of the most important limitations of the use of the PRV signal as surrogate of the HRV signal, as can be seen in Fig. 3(c).

In conclusion, our results indicate that there are some small differences in the time-varying spectral indices extracted from HRV and PRV, mainly in the HF band associated with respiration. Nevertheless, these differences were sufficiently small to suggest the use of the PRV signal as an alternative measurement of HRV signal during nonstationary conditions, at least during tilt table test.

\section{ACKNOWLEDGMENTS}

This work was partially supported by: Ministerio de Ciencia y Tecnología, FEDER under Project TEC2007-68076C02-02/TCM; and Diputación General de Aragón (DGA), Spain, through Grupos Consolidados GTC ref:T30.

\section{REFERENCES}

[1] Task Force, "Task Force of The European Society of Cardiology and The North American Society of Pacing and Electrophysiology. Heart rate variability: Standards of measurement, physiological interpretation, and clinical use," European Heart Journal, vol. 17, pp. 354-381, 1996.

[2] S. Lu, H. Zhao, K. Ju, K. Shin, M. Lee, K. Shelley, and K. Chon, "Can photoplethysmography variability serve as an alternative approach to obtain heart rate variability information?" Journal of Clinical Monitoring and Computing, vol. 22, pp. 23-29, 2008.

[3] J. Hayano, A. K. Barros, A. Kamiya, N. Ohte, and F. Yasuma, "Assessment of pulse rate variability by the method of pulse frequency demodulation," BioMedical Engineering OnLine, vol. 4, no. 62, 2005.

[4] K. Charlot, J. Cornolo, J. V. Brugniaux, J. P. Richalet, and A. Pichon, "Interchangeability between heart rate and photoplethysmography variabilities during sympathetic stimulations," Physiol. Meas., vol. 30, pp. 1357-1369, 2009.

[5] J. P. Martinez, R. Almeida, S. Olmos, A. P. Rocha, and P. Laguna, "A wavelet-based ECG delineator: Evaluation on standard databases," IEEE Trans. Biomed. Eng., vol. 51, no. 4, pp. 570-581, 2004.

[6] E. Gil, J. M. Vergara, and P. Laguna, "Detection of decreases in the amplitude fluctuation of pulse photoplethysmography signal as indication of obstructive sleep apnea syndrome in children," Biomed. Signal Process. Control, vol. 3, pp. 267-277, 2008.

[7] J. Mateo and P. Laguna, "Analysis of Heart Rate Variability in the Presence of Ectopic Beats Using the Heart Timing Signal," IEEE Trans. Biomed. Eng., vol. 50, no. 3, pp. 334-343, 2003.

[8] W. Martin and P. Flandrin, "Wigner-ville spectral analysis of nonstationary processes," Acoustics, Speech and Signal Processing, IEEE Transactions on, vol. 33, no. 6, pp. 1461-1470, 1985.

[9] M. Orini, R. Bailón, L. Mainardi, A. Minchol, and P. Laguna, "Continuous quantification of spectral coherence using quadratic timefrequency distributions: error analysis and application," Internat. Conf. Computers in Cardiology, pp. 681-684, 2009.

[10] I. Constant, D. Laude, I. Murat, and J. L. Elghozi, "Pulse rate variability is not a surrogate for heart rate variability," Clinical Science, vol. 97, pp. 391-397, 1999. 